\title{
Resveratrol induces apoptosis of human chronic myelogenous leukemia cells in vitro through p38 and JNK-regulated H2AX phosphorylation
}

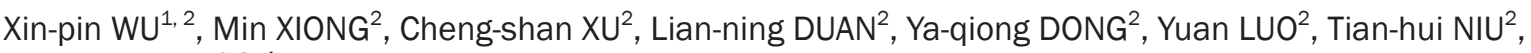 \\ Cheng-rong $\mathrm{LU}^{1,2, *}$ \\ ${ }^{1}$ Clinical School of Air Force General Hospital, Anhui Medical University, Heifei 230000, China; ${ }^{2}$ Aviation Medicine Research \\ Laboratory, Air Force General Hospital, PLA, Beijing 100142, China
}

\begin{abstract}
Aim: The phosphorylation of histone H2AX, a novel tumor suppressor protein, is involved in regulation of cancer cell apoptosis. The aim of this study was to examine whether $\mathrm{H} 2 \mathrm{AX}$ phosphorylation was required for resveratrol-induced apoptosis of human chronic myelogenous leukemia (CML) cells in vitro.

Methods: K562 cells were tested. Cell apoptosis was analyzed using flow cytometry, and the phosphorylation of H2AX and other signaling proteins was examined with Western blotting. To analyze the signaling pathways, the cells were transfected with lentiviral vectors encoding $\mathrm{H} 2 \mathrm{AX}$-wt or specific siRNAs.

Results: Treatment of K562 cells with resveratrol (20-100 $\mu \mathrm{mol} / \mathrm{L})$ induced apoptosis and phosphorylation of H2AX at Ser139 in time- and dose-dependent manners, but reduced phosphorylation of histone H3 at Ser10. Resveratrol treatment activated two MAPK family members p38 and JNK, and blocked the activation of another MAPK family member ERK. Pretreatment with the p38 inhibitor SB202190 or the JNK inhibitor SP600125 dose-dependently reduced resveratrol-induced phosphorylation of H2AX, which were also observed when the cells were transfected with p38- or JNK-specific siRNAs. Overexpression of H2AX in K562 cells markedly increased resveratrol-induced apoptosis, whereas overexpression of H2AX-139m (Ser139 was mutated to block phosphorylation) inhibited resveratrol-induced apoptosis. K562 cells transfected with $\mathrm{H} 2 \mathrm{AX}$-specific siRNAs were resistant to resveratrol-induced apoptosis. Conclusion: H2AX phosphorylation at Ser139 in human CML cells, which is regulated by p38 and JNK, is essential for resveratrolinduced apoptosis.
\end{abstract}

Keywords: resveratrol; chronic myelogenous leukemia; K562 cell; H2AX; apoptosis; p38; JNK; siRNA; SB202190; SP600125

Acta Pharmacologica Sinica (2015) 36: 353-361; doi: 10.1038/aps.2014.132; published online 26 Jan 2015

\section{Introduction}

Chronic myeloid leukemia (CML) is a clonal disease of hematopoietic stem cells that occurs because of a reciprocal translocation between chromosomes 9 and $22^{[1,2]}$. This chromosomal arrangement results in a $B C R-A B L$ fusion gene that encodes a BCR-ABL fusion oncoprotein, which possesses constitutively active tyrosine kinase activity and promotes leukemogenesis. Multiple signaling pathways are engaged by this abnormal BCR-ABL kinase, including Myc, Ras/mitogenactivated protein kinase (MAPK), c-Raf, MAPK/extracellular signal-regulated kinase (ERK), stress-activated protein kinase (SAPK)/JNK, signal transducer and activator of transcription (STAT), nuclear factor- $\mathrm{KB}$ (NF-kB), phosphatidylinositol

\footnotetext{
* To whom correspondence should be addressed.

E-mail luchengrong@263.net

Received 2014-07-29 Accepted 2014-10-15
}

3-kinase (PI3-K), c-Jun, c-cbl, CrkL, Janus kinase (Jak)/STAT and Src pathways ${ }^{[3,4]}$. Clinically, CML has been successfully treated with imatinib mesylate, which is a selective smallmolecule protein kinase inhibitor that specifically targets the oncogenic BCR-ABL fusion protein kinase ${ }^{[5-7]}$. However, $\sim 30 \%$ of CML patients develop intolerance or resistance to imatinib owing to a high frequency of point mutations or gene amplifications of the $B C R-A B L$ fusion gene ${ }^{[3]}$. Although some of these mutations can be overcome by treatment with next-generation tyrosine kinase inhibitors such as dasatinib or nilotinib, one mutation, T315I, causes complete insensitivity to all currently available tyrosine kinase inhibitors and is present in $\sim 15 \%$ of patients after failure of imatinib therapy ${ }^{[8-10]}$. More recently, other types of resistance that are independent of BCR-ABL and linked to the overexpression of the Src tyrosine kinases Lyn and Hck have also been demonstrated ${ }^{[11,12]}$. Thus, the search for novel and effective drugs against CML is an 
attractive aim.

Resveratrol (3,4',5-trans-trihydroxystilbene), which is a naturally polyphenolic phytoalexin, is found in $>70$ plant species, primarily in red grapes, red wine, peanuts, certain berries, dark chocolate, and other cocoa products ${ }^{[13,14]}$. Early research found that resveratrol elicits several beneficial effects related to human diseases, notably in the prevention of cardiovascular diseases $^{[15]}$. This compound is currently being used in Phase I studies to treat obesity and diabetes ${ }^{[16,17]}$. Recently, an increasing number of studies have revealed that resveratrol exhibits potent anti-cancer properties in certain epithelial tumors and leukemia ${ }^{[18,19]}$. Its potential chemopreventive and chemotherapeutic activities have been demonstrated in all three stages of carcinogenesis (initiation, promotion, and progression) in various murine models of human cancer ${ }^{[19]}$. Several in vitro studies have demonstrated that resveratrol can inhibit proliferation and induce apoptosis in various leukemic cell lines ${ }^{[20]}$. Resveratrol has also been demonstrated to induce apoptosis in B lineage leukemic cells (acute lymphoblastic leukemia) through the CD95-independent and mitochondria/caspase-9-specific pathway ${ }^{[21]}$. Another study has demonstrated that resveratrol induces the apoptosis of CML cells, including imatinib-resistant CML cells, in a caspase-independent manner ${ }^{[17]}$. Recently, Puissant et al found that resveratrol promotes autophagic cell death in CML cells ${ }^{[22]}$. Despite these promising in vitro and in vivo effects, resveratrol has not yet been analyzed as a potential therapeutic agent for the treatment of CML. To accelerate its clinical application, the detailed molecular mechanism of resveratrol against $\mathrm{CML}$ cells requires further clarification.

$\mathrm{H} 2 \mathrm{AX}$ is a variant of the histone H2A family. H2AX is known as a novel tumor suppressor protein owing to its role in the regulation of cancer cell apoptosis ${ }^{[23]}$. Our previous studies have demonstrated that H2AX phosphorylation at Ser139 is related to apoptosis in CML cells (K562 cells) ${ }^{[24,25]}$. In the current study, we used resveratrol to induce the apoptosis of K562 cells and to investigate the signaling pathways involved in the regulation of $\mathrm{H} 2 \mathrm{AX}$ phosphorylation. Finally, we evaluated the role of $\mathrm{H} 2 \mathrm{AX}$ phosphorylation in resveratrolinduced apoptosis of K562 cells, revealing the antileukemic effects of resveratrol. We demonstrated that resveratrol induced dramatic phosphorylation of $\mathrm{H} 2 \mathrm{AX}$ during apoptosis in a time- and dose-dependent manner. We also observed that the MAPK family members p38 and JNK were activated by resveratrol, which coincided with H2AX phosphorylation. The inhibition of p38 and JNK activity or the knockdown of p38 and JNK reduced H2AX phosphorylation. Overall, these data demonstrated that resveratrol-induced $\mathrm{H} 2 \mathrm{AX}$ phosphorylation in K562 cells is regulated by the p38 and JNK pathways. Furthermore, we provided evidence to confirm that H2AX phosphorylation at Ser139 is essential for the resveratrol-induced apoptosis of K562 cells.

\section{Materials and methods} Reagents

Resveratrol (trans-3,4',5-trihydroxystilbene), the p38 inhibitor SB202190 and the JNK inhibitor SP600125 were purchased from Sigma-Aldrich (St Louis, MO, USA). The antibodies against $\gamma \mathrm{H} 2 \mathrm{AX}$ (at Ser139), H2AX, caspase-3, cleaved caspase-3, phosphorylated JNK1/2 (pJNK1/2), total JNK1/2, phosphorylated ERK1/2 (pERK1/2), total ERK1/2, phosphorylated p38 (pp38), total p38, H3, phosphorylated H3 at Ser10 [pH3 (Ser10)], Bim, Bcl-2, and $\beta$-actin were obtained from Cell Signaling Technology (Beverly, MA, USA). H2AX siRNA was obtained from Santa Cruz Biotechnology (Santa Cruz, CA, USA), and p38- and JNK-specific siRNAs were obtained from Cell Signaling Technology.

\section{Lentivirus preparation}

HIV-based lentiviral expression plasmids, 293Ta lentiviral packaging cells and a Lenti-Pac HIV Expression Packaging Kit were obtained from GeneCopoeia (Rockville, MD, USA). At $48 \mathrm{~h}$ after seeding, the 293Ta packaging cells were transfected with lentiviral vectors encoding H2AX-wt (wild type; EX-B0074-LV201), H2AX-139m (Ser139 was mutated to block phosphorylation; CCS-B0074-LV201), or empty vector (EX-NEG-LV201) using a Lenti-Pac ${ }^{\mathrm{TM}}$ HIV Expression Packaging Kit according to the manufacturer's instructions. The lentiviruses were harvested by collecting the pseudoviruscontaining culture medium at $48 \mathrm{~h}$ post-transfection. K562 cells were transduced with the harvested lentiviral particles in the presence of $5 \mu \mathrm{g} / \mathrm{mL}$ polybrene (Santa Cruz Biotechnology) and cultured in selection medium with 2 $\mu \mathrm{g} / \mathrm{mL}$ puromycin for $\sim 2$ weeks to allow the cells to grow to $90 \%$ confluence.

\section{siRNA transfection}

To knock down the endogenous expression levels of H2AX, p38, or JNK, siRNAs targeting H2AX, p38, or JNK were transfected into K562 cells according to the manufacturer's instructions, and the effect of knockdown was assessed by Western blotting. K562 cells transfected with control siRNA served as controls.

\section{Cell culture and drug treatment}

K562 cells were cultured in RPMI-1640 medium supplemented with $10 \%$ fetal bovine serum in a $37^{\circ} \mathrm{C}, 5 \% \mathrm{CO}_{2}$ incubator. Before each experiment, K562 cells, K562 cells transfected with siRNAs targeting p38, JNK, or H2AX, and stable K562 cells transduced with the lentiviral particles (overexpression of H2AX-wt and H2AX-139m) were seeded at $1 \times 10^{6}$ cells $/ \mathrm{mL}$ in $10-\mathrm{cm}$ dishes and treated with resveratrol, p38 inhibitor SB202190, or JNK inhibitor SP600125 $1 \mathrm{~h}$ before resveratrol treatment.

\section{Whole cellular protein or histone extraction and Western blot analysis}

Whole cellular proteins were extracted after drug treatment by disrupting the cells in lysis buffer $(50 \mathrm{mmol} / \mathrm{L}$ Tris-HCI, $\mathrm{pH} 7.4,1 \% \mathrm{NP}-40,0.25 \%$ sodium deoxycholate, $150 \mathrm{mmol} / \mathrm{L}$ $\mathrm{NaCl}, 1 \mathrm{mmol} / \mathrm{L}$ EGTA, $1 \mathrm{mmol} / \mathrm{L} \mathrm{Na}_{3} \mathrm{VO}_{4}, 1 \mathrm{mmol} / \mathrm{L} \mathrm{NaF}$, $1 \mathrm{mg} / \mathrm{mL}$ aprotinin, $1 \mathrm{mg} / \mathrm{mL}$ leupeptin, $1 \mathrm{mg} / \mathrm{mL}$ pepstatin, and $1 \mathrm{mmol} / \mathrm{L} \mathrm{PMSF}$ ). To extract the histones after drug treat- 
ment, the cells were lysed in NETN buffer $(150 \mathrm{mmol} / \mathrm{L} \mathrm{NaCl}$, $1 \mathrm{mmol} / \mathrm{L}$ EDTA, $20 \mathrm{mmol} / \mathrm{L}$ Tris, $\mathrm{pH} 8$, and $0.5 \% \mathrm{NP}-40$ ) and centrifuged for $5 \mathrm{~min}^{[26]}$. The histones were extracted from the pellets with $0.1 \mathrm{~mol} / \mathrm{L} \mathrm{HCl}$. The protein samples were resolved by SDS-PAGE and transferred to polyvinylidene difluoride membranes. The membranes were blocked at room temperature for $1 \mathrm{~h}$ with $5 \%$ nonfat milk or BSA in Tris-buffered saline containing Tween 20 (TBST). Primary antibodies were incubated with the membranes in TBST with 5\% nonfat milk or BSA at $4{ }^{\circ} \mathrm{C}$ overnight. The membranes were incubated with the appropriate secondary antibody in TBST with $5 \%$ nonfat milk or BSA for $1 \mathrm{~h}$ at room temperature. Proteins were detected using SignalFire ECL Reagent (Cell Signaling Technology).

\section{Flow cytometry}

After drug treatment, K562 cells $\left(1 \times 10^{6}\right)$ were washed with $1 \times$ binding buffer at room temperature. The cells were incubated with Annexin V for 15 min. The cells were then incubated with propidium iodide (PI) before detection, followed by analysis with flow cytometry (FACS-Calibur; BectonDickinson, San Jose, CA, USA). All reagents were obtained from an Apoptosis Detection Kit (BD Biosciences, San Jose, CA, USA).

\section{Statistical analysis}

The data are expressed as the mean $\pm \mathrm{SD}$ of three independent determinations, and Student's t-test or ANOVA was performed using SPSS version 17.0 software (SPSS INC, Chicago, IL, USA). The significance levels of all tests were set at $P<0.05$.

\section{Results}

Resveratrol induces H2AX phosphorylation and apoptosis in K562 cells

Previous data, including our own, have demonstrated that histone H2AX phosphorylation at Ser139 is involved in apoptotic regulation ${ }^{[26]}$. In particular, $\mathrm{H} 2 \mathrm{AX}$ phosphorylation at Ser139 has an important role in the apoptosis of tumor cells $^{[24,25,27]}$. Hence, we investigated whether resveratrol could induce H2AX phosphorylation in CML cells. Histone samples were extracted from K562 cells after resveratrol treatment at the indicated time and dose, and Western blot analysis was conducted. Resveratrol treatment for $48 \mathrm{~h}$ induced dramatic phosphorylation of $\mathrm{H} 2 \mathrm{AX}(\gamma \mathrm{H} 2 \mathrm{AX})$ in a dose-dependent manner (Figure 1A). Resveratrol $(60 \mu \mathrm{mol} / \mathrm{L})$ treatment also induced strong phosphorylation of H2AX in a timedependent manner (Figure 1A). $\mathrm{\gamma H} 2 \mathrm{AX}$ was observed as early as $24 \mathrm{~h}$ after resveratrol treatment and was maintained for up to $72 \mathrm{~h}$. We also observed that resveratrol strongly inhibited histone $\mathrm{H} 3$ phosphorylation at Ser10 (Figure 1A). The detailed mechanism involved in resveratrol-reduced H3 phosphorylation and its function requires further study.

Resveratrol was previously reported to induce apoptosis in CML cells ${ }^{[17]}$. To test this, we treated $\mathrm{K} 562$ cells with resveratrol at the indicated time and detected apoptosis with flow cytometry. Resveratrol stimulated K562 cell apoptosis in a time-dependent manner (Figure 1B), following a parallel response with H2AX phosphorylation (Figure 1A). The percentage of total apoptotic cells (including early and late apoptotic cells) after treatment with resveratrol for $72 \mathrm{~h}$ reached $>90 \%$ (Figure 1B). These data demonstrated that resveratrol induced $\mathrm{H} 2 \mathrm{AX}$ phosphorylation during the apoptosis of K562 cells, suggesting that $\mathrm{H} 2 \mathrm{AX}$ phosphorylation may be involved in regulating resveratrol-induced apoptosis in CML cells.

\section{Resveratrol stimulates p38 and JNK activation in K562 cells during apoptosis}

The MAPK family (ERK, p38, and JNK) has been implicated in many human tumors. Therefore, the MAPK pathways have become a focus of intense investigation for developing small-molecule inhibitors as targeted therapeutics ${ }^{[28]}$. Our previous data demonstrated that p38 MAPK is involved in imatinib-induced apoptosis in CML cells ${ }^{[25]}$. In the current study, we investigated the role of MAPK pathways (ERK, p38, and JNK) in apoptosis and in H2AX phosphorylation in CML cells induced by resveratrol. K562 cells were incubated with resveratrol for the indicated time, and whole cellular proteins were extracted for Western blot analysis. Resveratrol induced p38 and JNK phosphorylation in a time-dependent manner (Figure 2), following a response similar to that for H2AX phosphorylation (Figure 1A). Unexpectedly, resveratrol strongly inhibited ERK phosphorylation (Figure 2), which is activated by BCR-ABL with constitutively active tyrosine kinase activity in CML cells ${ }^{[25]}$. This finding is not consistent with the results reported by Mustafi et al, indicating that resveratrol stimulates ERK activity in K562 cells ${ }^{[29]}$. Additionally, we used the whole cellular proteins extracted from resveratrol-treated K562 cells to analyze apoptosis with Western blotting. Resveratrol increased caspase- 3 cleavage to form cleaved caspase-3 (active caspase-3), which is a hallmark of apoptosis (Figure 2). We also observed that the expression of another potent proapoptotic protein, Bim (B-cell lymphoma 2 interacting mediator of cell death), was increased by resveratrol treatment. However, the level of Bcl-2 (B-cell lymphoma2), which exerts an antiapoptotic effect, was not affected by resveratrol treatment (Figure 2). These results, together with those discussed above (Figure 1), indicated that resveratrol treatment triggered p38 and JNK activation, followed by H2AX phosphorylation in K562 cells during apoptosis, suggesting that p38 and JNK are involved in regulating $\mathrm{H} 2 \mathrm{AX}$ phosphorylation.

\section{p38 and JNK regulate resveratrol-induced H2AX phosphorylation in $\mathrm{K} 562$ cells}

We investigated whether p38 and JNK are actually involved in regulating $\mathrm{H} 2 \mathrm{AX}$ phosphorylation. K562 cells were preincubated with the p38 inhibitor SB202190 or with the JNK inhibitor SP600125 before resveratrol treatment, and the $\gamma \mathrm{H} 2 \mathrm{AX}$ level was analyzed by Western blotting. p38 and JNK phosphorylation was also detected to confirm the effects of SB202190 and SP600125 on their targeted kinases. Treatment 
A

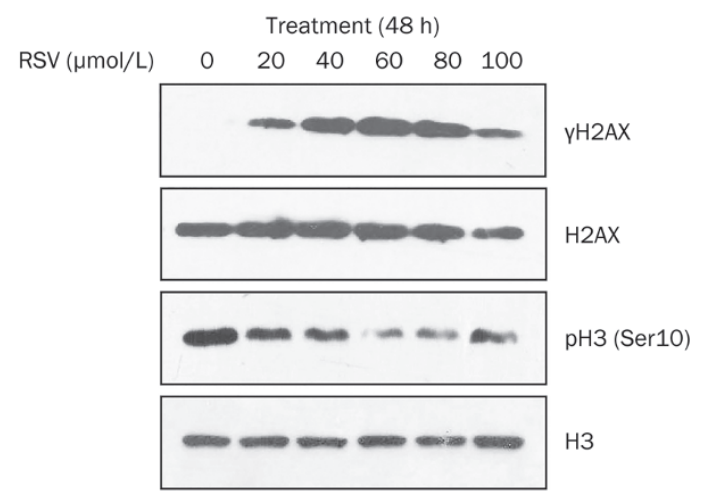

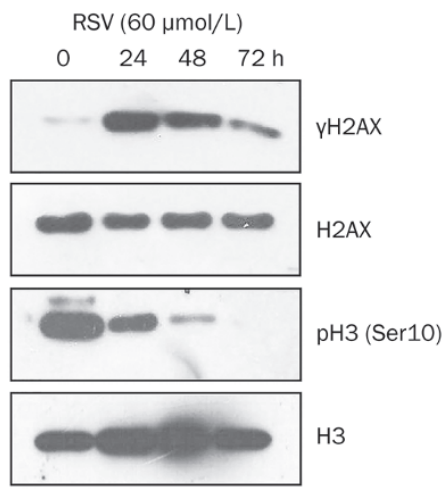

B

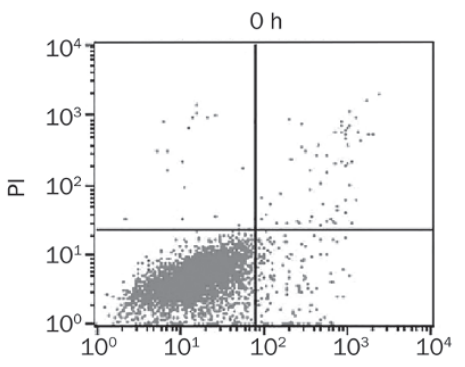

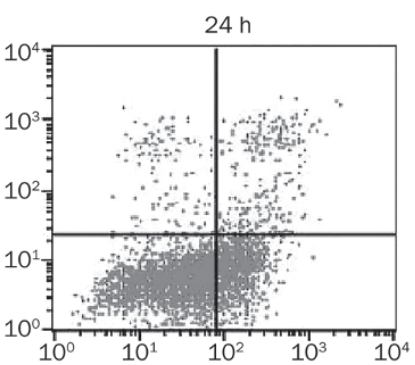

$\operatorname{RSV}(60 \mu \mathrm{mol} / \mathrm{L})$
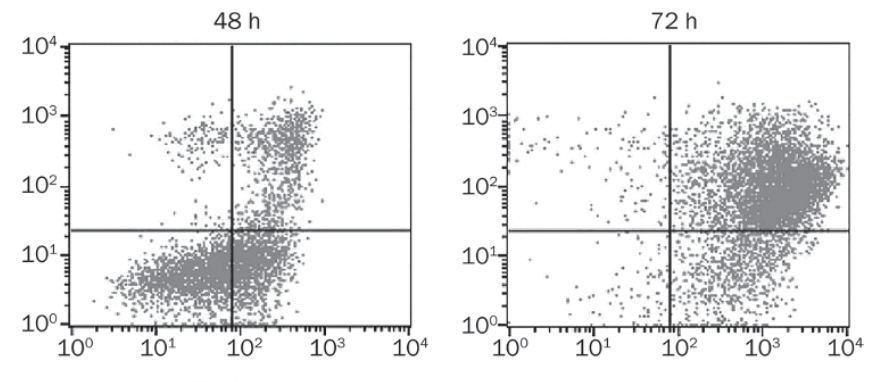

Annexin V FITC

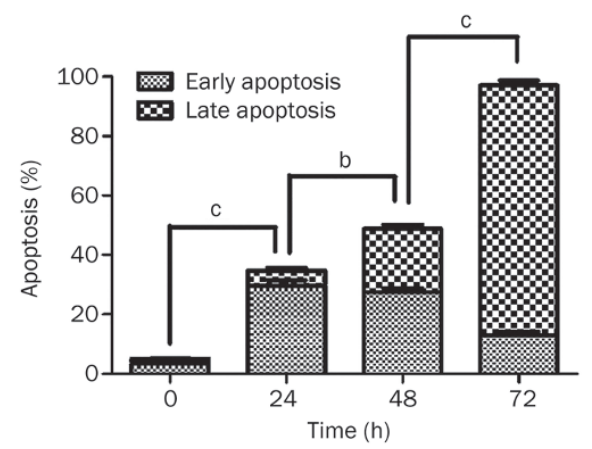

Figure 1. Resveratrol induces $\mathrm{H} 2 \mathrm{AX}$ phosphorylation and apoptosis. (A) K562 cells were treated with different concentrations of resveratrol (RSV) for $48 \mathrm{~h}$ (left panel) or treated with $60 \mu \mathrm{mol} / \mathrm{L}$ resveratrol for different time periods (right panel). Cells not treated with resveratrol served as negative controls. The histone samples were extracted for Western blot analysis using antibodies against $\mathrm{YH} 2 \mathrm{AX}$ (phosphorylated $\mathrm{H} 2 \mathrm{AX}$ at Ser139), total H2AX, pH3 (Ser10) (phosphorylated H3 at Ser10), and total H3. (B) K562 cells were treated with $60 \mu \mathrm{mol} / \mathrm{L}$ resveratrol. Cells were harvested at the indicated time and then subjected to flow cytometry after Annexin V/PI staining to assess apoptosis (upper panel). The mean \pm SD of three independent determinations is shown (lower panel). ${ }^{b} P<0.05,{ }^{c} P<0.01$.

with the p38 inhibitor SB202190 or with the JNK inhibitor SP600125 inhibited p38 or JNK activity, respectively, and at the same time dramatically suppressed resveratrol-induced $\mathrm{H} 2 \mathrm{AX}$ phosphorylation (level of $\gamma \mathrm{H} 2 \mathrm{AX}$ ) (Figure 3A and 3B). To definitively establish the roles of the p38 and JNK signaling pathways in H2AX phosphorylation, we used p38- and JNKspecific siRNAs to inhibit p38 and JNK expression, respectively, and analyzed the effects of p38 and JNK knockdown on resveratrol-induced $\mathrm{H} 2 \mathrm{AX}$ phosphorylation. Consistent with the findings using SB202190 and SP600125 in K562 cells, transfection with p38- and JNK-specific siRNAs, but not with control siRNA, inhibited resveratrol-induced H2AX phosphorylation (Figure 3C). p38- and JNK-specific siRNAs were each confirmed to suppress the expression of their respective targeted kinases (Figure 3C). These data demonstrated that p38 and JNK regulate resveratrol-induced $\mathrm{H} 2 \mathrm{AX}$ phosphorylation in K562 cells.

$\mathrm{H} 2 \mathrm{AX}$ phosphorylation is required for the resveratrol-induced apoptosis of K562 cells

We have previously reported that H2AX phosphorylation has a critical role in apoptotic regulation ${ }^{[26]}$. Apoptosis is almost completely blocked in $\mathrm{H} 2 \mathrm{AX}^{-/-}$mouse embryo fibroblasts (MEFs). The transfection of H2AX-wt, but not H2AX-139m (Ser139 was mutated to block H2AX phosphorylation), into $\mathrm{H} 2 \mathrm{AX}^{-/-} \mathrm{MEFs}$ restored the ability to 

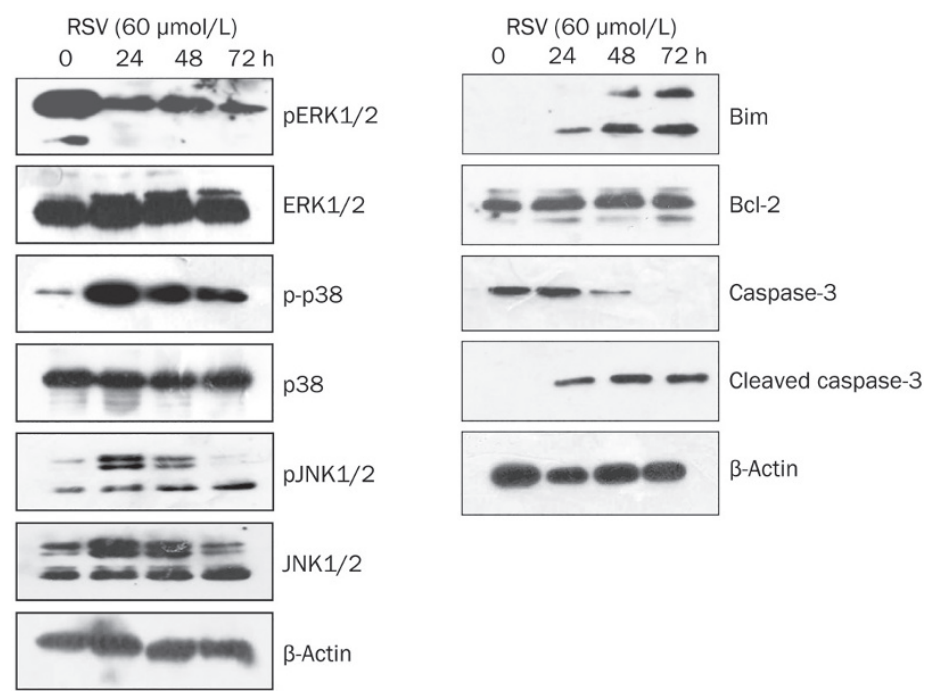

Figure 2. Resveratrol triggers p38 and JNK activation during apoptosis. K562 cells were treated with $60 \mu \mathrm{mol} / \mathrm{L}$ resveratrol for the indicated time. The extracted whole proteins were used for Western blot analysis with antibodies against ERK1/2, pERK1/2, p38, p-p38, JNK1/2, and pJNK1/2 (left panel), as well as against caspase-3, cleaved caspase-3, Bim, and Bcl-2 (right panel). $\beta$-Actin was detected as a loading control.

induce apoptosis. Increasing evidence has also demonstrated that H2AX phosphorylation is involved in the apoptosis of tumor cells ${ }^{[23,25,27]}$. To investigate the important role of $\gamma \mathrm{H} 2 \mathrm{AX}$ in the resveratrol-induced apoptosis of CML cells, we assessed the effects of H2AX overexpression and knockdown on the apoptosis of CML cells. The stable K562 cell lines transduced with either the lentiviral vectors encoding H2AX-wt or H2AX-139m, or the empty vector control were used to detect resveratrol sensitivity. After $24 \mathrm{~h}$ of continuous treatment with resveratrol, the above cells were analyzed by flow cytometry for apoptosis. The stable K562 cells without resveratrol treatment served as controls (Figure 4A). The data demonstrated that H2AX-wt overexpression strongly increased K562 cell apoptosis (Figure 4A). The percentage of apoptotic K562 cells harboring empty vectors reached only $40 \%$ (including early and late apoptosis) after resveratrol treatment; however, the percentage of apoptotic K562 cells overexpressing $\mathrm{H} 2 \mathrm{AX}-\mathrm{wt}$ reached almost $90 \%$ after the same treatment (Figure 4A). By contrast, the K562 cells with H2AX-139m overexpression were resistant to resveratrolinduced apoptosis, and the percentage of apoptotic cells reached $30 \%$, which is a lower percentage than that of K562 cells harboring empty vectors (Figure 4A). These results were also confirmed by Western blot analysis (Figure 4B). $\mathrm{H} 2 \mathrm{AX}$-wt overexpression increased the resveratrol-induced expression of Bim compared with that of the empty vector control (Figure 4B). H2AX-139m overexpression inhibited the resveratrol-induced expression of Bim compared with that of the empty vector control or $\mathrm{H} 2 \mathrm{AX}-\mathrm{wt}$ overexpression (Figure 4B). Consistent with the above data (Figure 2), Bcl-2 expression levels did not differ between these stable K562 cells (Figure 4B). Seemingly, H2AX-139m overexpression reduced endogenous H2AX (Ser139) phosphorylation and inhibited resveratrol-induced apoptosis in K562 cells. Furthermore,
H2AX knockdown in K562 cells transfected with H2AX-specific siRNA strongly inhibited resveratrol-induced apoptosis. The apoptotic percentage of K562 cells with H2AX knockdown decreased from $38 \%$, the apoptotic percentage of K562 cells transfected with control siRNA, to 10\% (Figure 5A). H2AX knockdown also blocked resveratrol-induced Bim expression (Figure 5B). These findings demonstrate that H2AX phosphorylation is required for the apoptosis of CML cells.

\section{Discussion}

Resveratrol has been demonstrated to induce apoptosis in CML cells, including imatinib-resistant CML cells, suggesting that this compound could act against CML by bypassing the $\mathrm{BCR}-\mathrm{ABL}$ fusion protein, which possesses constitutively active tyrosine kinase activity and promotes leukemogenesis. In this study, we found a novel mechanism to be involved in the antileukemic effect of resveratrol. The resveratrol-induced apoptosis of K562 cells is related to the phosphorylation of the tumor suppressor protein H2AX at Ser139. Resveratrol treatment stimulated $\mathrm{H} 2 \mathrm{AX}$ phosphorylation and apoptosis in $\mathrm{K} 562$ cells (Figure 1). H2AX overexpression in K562 cells increased resveratrol-induced apoptosis; however, overexpression of Ser139-mutated H2AX (which blocks phosphorylation) resulted in K562 cells that were resistant to apoptotic induction with resveratrol (Figure 4). These data demonstrated that H2AX phosphorylation is essential for resveratrol-induced apoptosis. This conclusion was confirmed in K562 cells with H2AX knockdown. Compared with control siRNA, H2AX-specific siRNA strongly reduced resveratrolinduced apoptosis (Figure 5), which is consistent with the finding in K562 cells overexpressing H2AX-139m (Figure 4). The above results indicated that histone $\mathrm{H} 2 \mathrm{AX}$ can serve as a novel and important molecule that can be targeted by resveratrol.

In searching for the upstream signaling pathways that regu- 
A

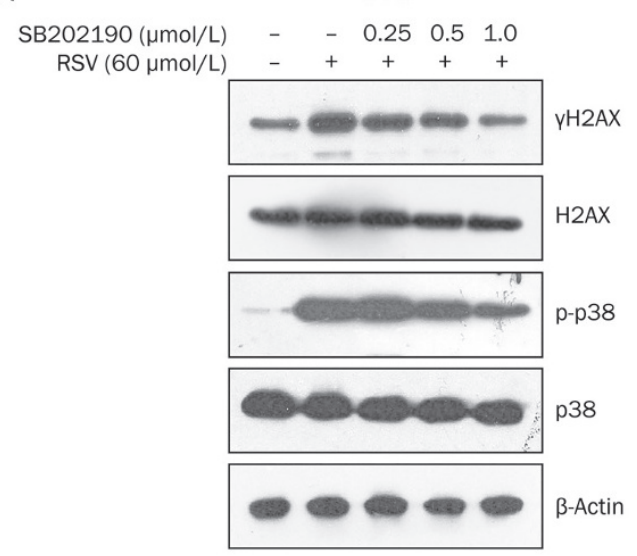

B

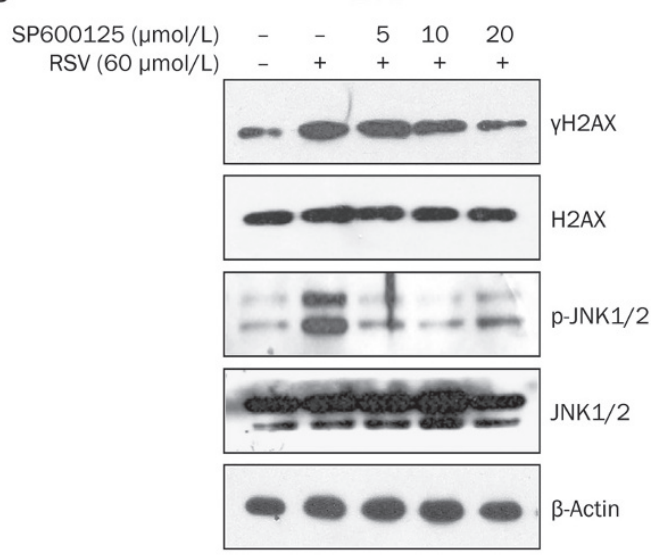

C

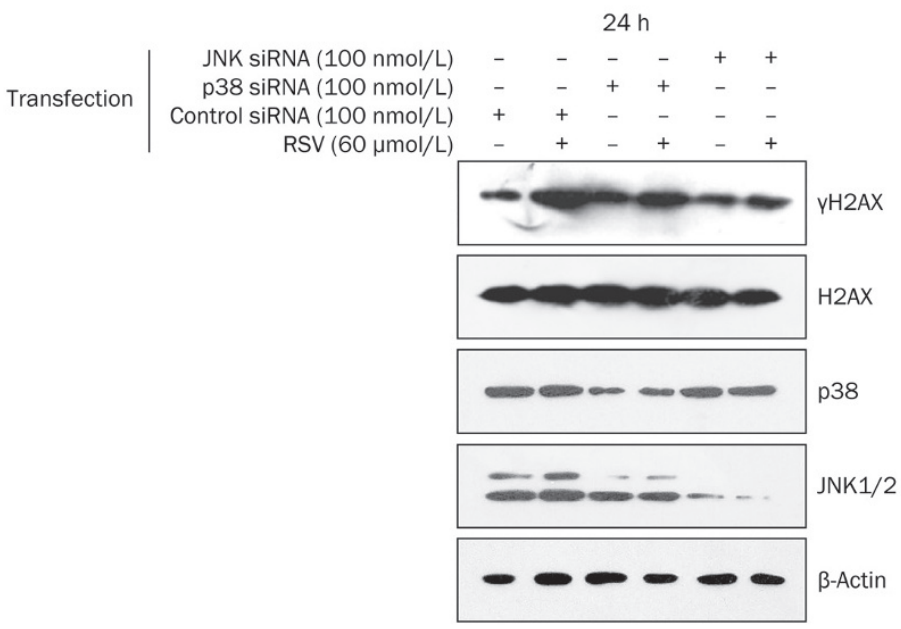

Figure 3. p38 and JNK1/2 pathways regulate resveratrol-induced H2AX phosphorylation. (A) K562 cells were treated with $60 \mu$ mol/L resveratrol, which was combined with the indicated concentration of the p38 inhibitor SB202190, for $24 \mathrm{~h}$. The extracted whole cellular protein samples were subjected to Western blot analysis with antibodies against p-p38 and total p38, and the histone samples were used for Western blot analysis with antibodies against $\mathrm{yH} 2 \mathrm{AX}$ and total $\mathrm{H} 2 \mathrm{AX}$. (B) $\mathrm{K} 562$ cells were treated with $60 \mu \mathrm{mol} / \mathrm{L}$ resveratrol, which was combined with the indicated concentration of the JNK inhibitor SP600125, for $24 \mathrm{~h}$. The whole cellular protein samples were evaluated for $\mathrm{pJNK} 1 / 2$ and total JNK1/2, and the histone protein samples were evaluated for $\mathrm{YH} 2 \mathrm{AX}$ and total $\mathrm{H} 2 \mathrm{AX}$. B-Actin was used to confirm equal whole cellular protein loading. (C) After being transfected with p38-specific siRNA (100 nmol/L), JNK-specific siRNA (100 nmol/L) or control siRNA (100 nmol/L), the K562 cells were treated with $60 \mu \mathrm{mol} / \mathrm{L}$ resveratrol for $24 \mathrm{~h}$. The histone samples were extracted to detect $\mathrm{yH} 2 \mathrm{AX}$ and total $\mathrm{H} 2 \mathrm{AX}$, and the extracted whole cellular protein samples were used to detect the effects of p38 and JNK knockdown. $\beta$-Actin was used to confirm equal whole cellular protein loading.

late $\mathrm{H} 2 \mathrm{AX}$ phosphorylation, we found that resveratrol could activate p38 and JNK. When these two kinases were inhibited by their inhibitors or by siRNA for knockdown, H2AX phosphorylation was also inhibited (Figure 3). Overall, these data demonstrated that the p38 and JNK pathways regulate H2AX phosphorylation that is induced by resveratrol in K562 cells. Previously, we reported that UVA radiation induces H2AX phosphorylation by the JNK pathway in MEFs and that imatinib induces $\mathrm{H} 2 \mathrm{AX}$ phosphorylation by the p38 pathway in $\mathrm{K} 562$ cells $^{[25,26]}$. The current study revealed that resveratrol triggered both the $\mathrm{p} 38$ and JNK pathways, which are involved in H2AX phosphorylation. These findings demonstrated that the signaling network regulating $\mathrm{H} 2 \mathrm{AX}$ phosphorylation is complex. We also found that another MAPK family member, ERK, was strongly activated in K562 cells, characterized by high levels of phosphorylated ERK1/2 (Figure 2). Unexpectedly, resveratrol treatment could dramatically block ERK1/2 phosphorylation in K562 cells (Figure 2). The role of ERK inhibition by resveratrol in the regulation of $\mathrm{K} 562$ cell function is interesting and requires further investigation.

\section{Acknowledgements}

This work was supported by the National Natural Science Foundation of China (№ 81070424 and № 81272303).

\section{Author contribution}

Xin-pin WU and Min XIONG performed most of the experiments. Cheng-rong LU and Lian-ning DUAN designed the study and analyzed the results. Cheng-rong LU wrote the manuscript, which was revised by Lian-ning DUAN. Cheng- 
A

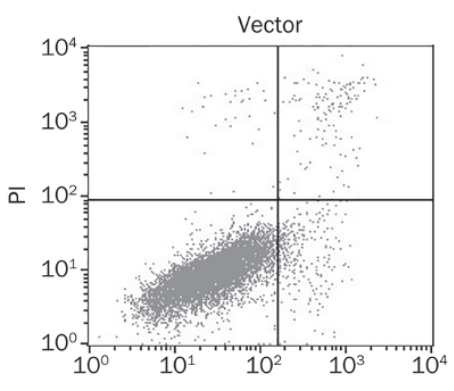

RSV $(60 \mu \mathrm{mol} / \mathrm{L})$ treatment for $0 \mathrm{~h}$
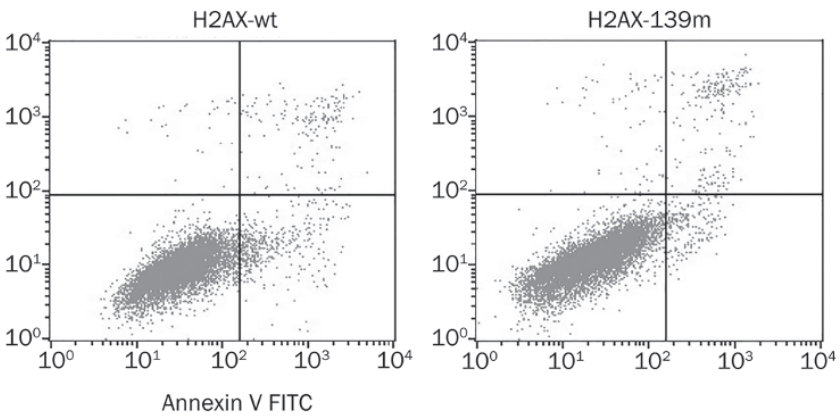

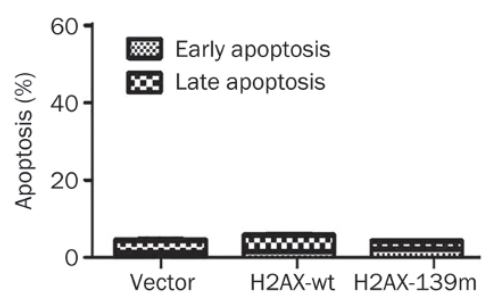

RSV (60 $\mu \mathrm{mol} / \mathrm{L})$ treatment for $24 \mathrm{~h}$
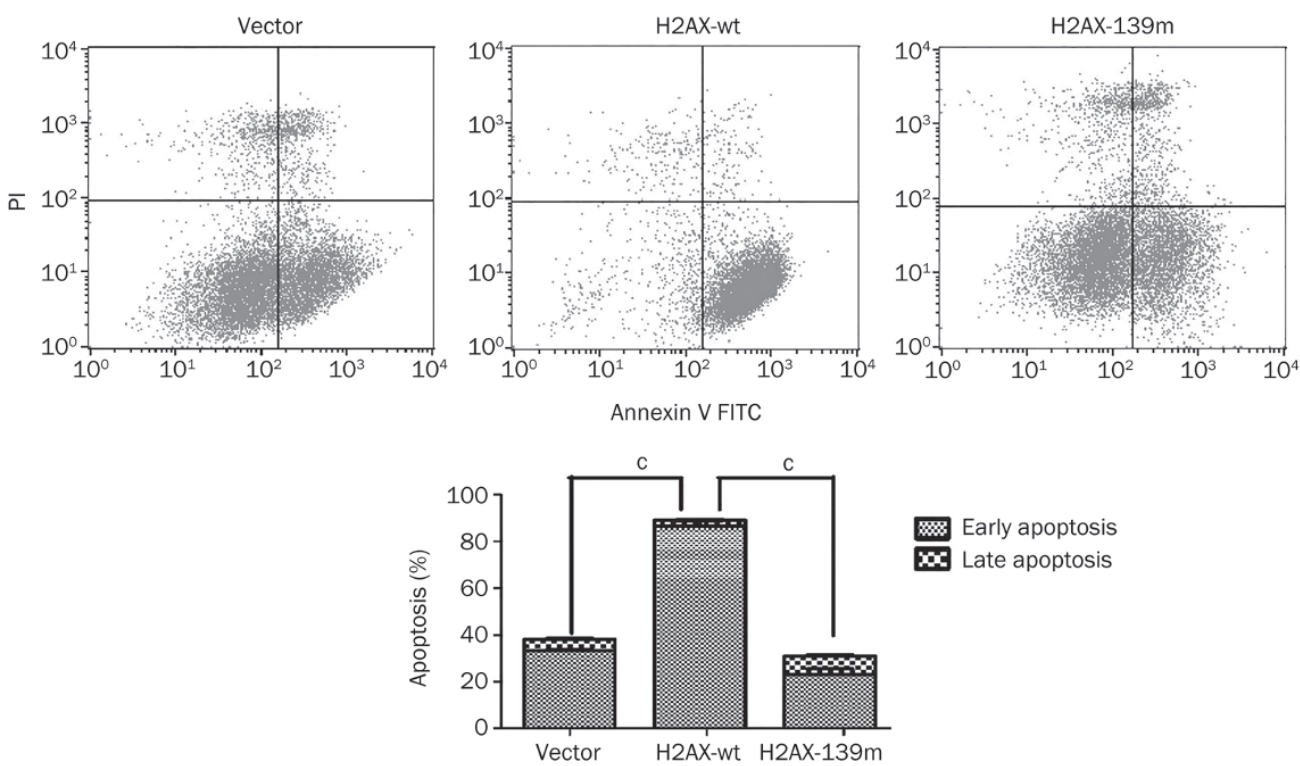

B

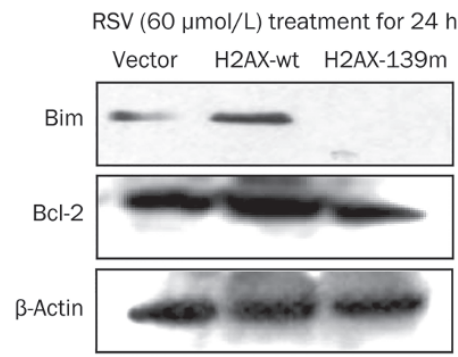

Figure 4. H2AX phosphorylation increases resveratrol-induced apoptosis. (A) K562 cells were transfected with lentiviral vectors encoding $\mathrm{H} 2 \mathrm{AX}$-wt, H2AX-139m (blocking Ser139 phosphorylation) or the empty vector alone control. After selection, the stable cells were treated with 60 umol/L resveratrol for 0 and $24 \mathrm{~h}$ and subjected to flow cytometry for the analysis of apoptotic cells (early and late apoptosis). (B) The stable K562 cells harboring vector encoding $\mathrm{H} 2 \mathrm{AX}$-wt or $\mathrm{H} 2 \mathrm{AX}-139 \mathrm{~m}$ were treated with $60 \mu \mathrm{mol} / \mathrm{L}$ resveratrol for $24 \mathrm{~h}$ and then harvested for Western blot analysis with antibodies against Bim and $\mathrm{Bcl}-2$. $\beta$-Actin was used to confirm equal whole cellular protein loading. The mean $\pm \mathrm{SD}$ of three independent determinations are shown. ${ }^{\mathrm{C}} P<0.01$. 
A
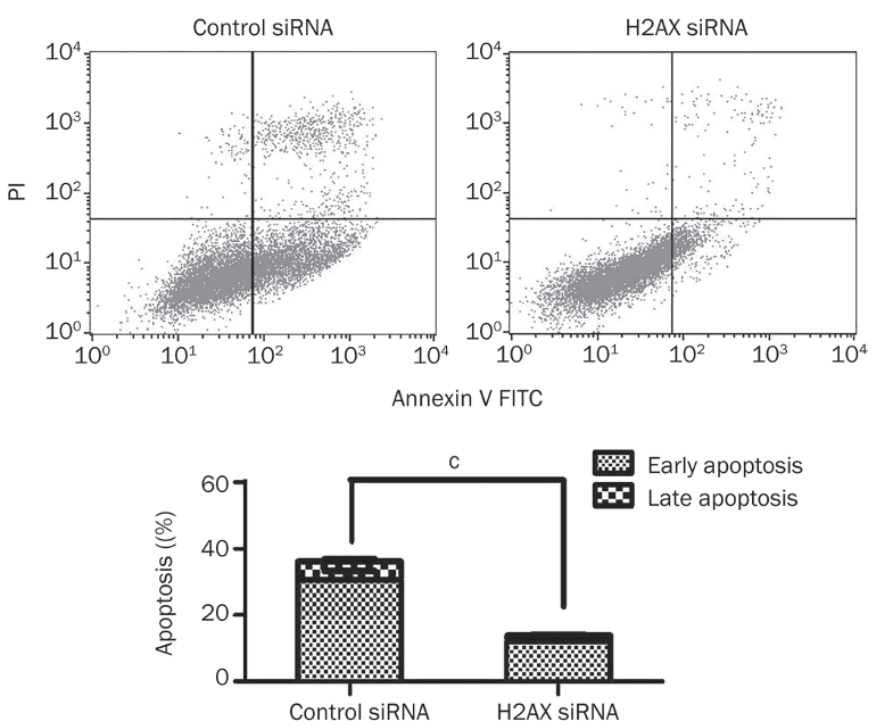

B

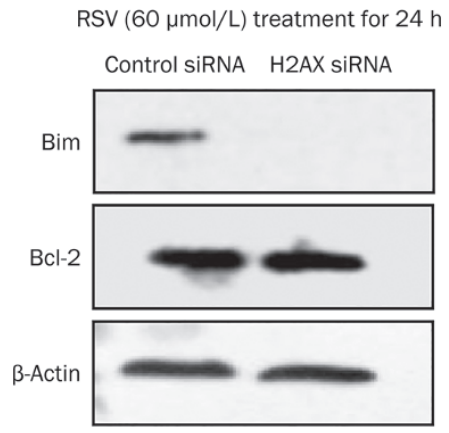

Figure 5. H2AX knockdown inhibits resveratrol-induced apoptosis. (A) To detect the effect of $\mathrm{H} 2 \mathrm{AX}$ phosphorylation on resveratrol-induced apoptosis, $\mathrm{K} 562$ cells were transfected with $\mathrm{H} 2 \mathrm{AX}$-specific siRNA or control siRNA as a negative control. The transfected cells were treated with 60 $\mu \mathrm{mol} / \mathrm{L}$ resveratrol for $24 \mathrm{~h}$ and subjected to flow cytometry for analysis of apoptotic cells (early and late apoptosis). (B) K562 cells transfected with H2AX-specific siRNA or control siRNA were treated with $60 \mu \mathrm{mol} / \mathrm{L}$ resveratrol for $24 \mathrm{~h}$ and then harvested for Western blot analysis with antibodies against Bim and $\mathrm{Bcl}-2$. $\beta$-Actin was used to confirm equal whole cellular protein loading. The mean \pm SD of three independent determinations are shown. ${ }^{\circ} P<0.01$.

shan XU, Ya-qiong DONG, and Yuan LUO performed the flow cytometry analysis for apoptosis. Tian-hui NIU also provided support and general guidance for this work.

\section{References}

1 Goldman JM, Melo JV. Chronic myeloid leukemia--advances in biology and new approaches to treatment. N Engl J Med 2003; 349: 145164.

2 Goldman JM, Melo JV. Targeting the BCR-ABL tyrosine kinase in chronic myeloid leukemia. N Engl J Med 2001; 344: 1084-6.

3 Dumka D, Puri P, Carayol N, Lumby C, Balachandran H, Schuster K, et al. Activation of the p38 Map kinase pathway is essential for the antileukemic effects of dasatinib. Leuk Lymphoma 2009; 50: 2017 -
29.

4 Horita M, Andreu EJ, Benito A, Arbona C, Sanz C, Benet I, et al. Blockade of the Bcr-Abl kinase activity induces apoptosis of chronic myelogenous leukemia cells by suppressing signal transducer and activator of transcription 5-dependent expression of Bcl-xL. J Exp Med 2000; 191: 977-84.

5 Branford S, Yeung DT, Parker WT, Roberts ND, Purins L, Braley JA, et al. Prognosis for patients with CML and $>10 \%$ BCR-ABL1 after 3 months of imatinib depends on the rate of BCR-ABL1 decline. Blood 2014; 124: 511-8.

6 Chu S, Holtz M, Gupta M, Bhatia R. BCR/ABL kinase inhibition by imatinib mesylate enhances MAP kinase activity in chronic myelogenous leukemia CD34 ${ }^{+}$cells. Blood 2004; 103: 3167-74.

7 Lu Z, Jin Y, Chen C, Li J, Cao Q, Pan J. Pristimerin induces apoptosis in imatinib-resistant chronic myelogenous leukemia cells harboring T315I mutation by blocking NF-kappaB signaling and depleting BcrAbl. Mol Cancer 2010; 9: 112.

8 Mesa RA. Imatinib and tyrosine kinase inhibition, in the management of BCR-ABL negative myeloproliferative disorders. Biologics 2007; 1 : 129-38.

9 Deguchi Y, Kimura S, Ashihara E, Niwa T, Hodohara K, Fujiyama Y, et al. Comparison of imatinib, dasatinib, nilotinib and INNO-406 in imatinib-resistant cell lines. Leuk Res 2008; 32: 980-3.

10 Nicolini FE, Ibrahim AR, Soverini S, Martinelli G, Muller MC, Hochhaus A, et al. The BCR-ABLT315I mutation compromises survival in chronic phase chronic myelogenous leukemia patients resistant to tyrosine kinase inhibitors, in a matched pair analysis. Haematologica 2013; 98: 1510-6.

11 Donato NJ, Wu JY, Stapley J, Gallick G, Lin H, Arlinghaus R, et al. BCR-ABL independence and LYN kinase overexpression in chronic myelogenous leukemia cells selected for resistance to STI571. Blood 2003; 101: 690-8.

$12 \mathrm{Hu}$ Y, Liu Y, Pelletier S, Buchdunger E, Warmuth M, Fabbro D, et al. Requirement of Src kinases Lyn, Hck and Fgr for BCR-ABL1-induced B-lymphoblastic leukemia but not chronic myeloid leukemia. Nat Genet 2004; 36: 453-61.

13 Athar M, Back JH, Tang X, Kim KH, Kopelovich L, Bickers DR, et al. Resveratrol: a review of preclinical studies for human cancer prevention. Toxicol Appl Pharmacol 2007; 224: 274-83.

14 Li F, Gong Q, Dong H, Shi J. Resveratrol, a neuroprotective supplement for Alzheimer's disease. Curr Pharm Des 2012; 18: 27-33.

15 Shen M, Jia GL, Wang YM, Ma H. Cardioprotective effect of resvaratrol pretreatment on myocardial ischemia-reperfusion induced injury in rats. Vascul Pharmacol 2006; 45: 122-6.

16 Park SJ, Ahmad F, Philp A, Baar K, Williams T, Luo H, et al. Resveratrol ameliorates aging-related metabolic phenotypes by inhibiting CAMP phosphodiesterases. Cell 2012; 148: 421-33.

17 Puissant A, Grosso S, Jacquel A, Belhacene N, Colosetti P, Cassuto $\mathrm{JP}$, et al. Imatinib mesylate-resistant human chronic myelogenous leukemia cell lines exhibit high sensitivity to the phytoalexin resveratrol. Faseb J 2008; 22: 1894-904.

18 Surh YJ, Hurh YJ, Kang JY, Lee E, Kong G, Lee SJ. Resveratrol, an antioxidant present in red wine, induces apoptosis in human promyelocytic leukemia (HL-60) cells. Cancer Lett 1999; 140: 1-10.

19 Whyte L, Huang YY, Torres K, Mehta RG. Molecular mechanisms of resveratrol action in lung cancer cells using dual protein and microarray analyses. Cancer Res 2007; 67: 12007-17.

$20 \mathrm{Li} \mathrm{T}$, Wang W, Li T. Antileukemic mechanism of resveratrol in vitro and in mice bearing L1210-tumor. Zhong Xi Yi Jie He Xue Bao 2008; 6: 1267-74.

21 Dorrie J, Gerauer H, Wachter Y, Zunino SJ. Resveratrol induces 
extensive apoptosis by depolarizing mitochondrial membranes and activating caspase-9 in acute lymphoblastic leukemia cells. Cancer Res 2001; 61: 4731-9.

22 Puissant A, Robert G, Fenouille N, Luciano F, Cassuto JP, Raynaud $\mathrm{S}$, et al. Resveratrol promotes autophagic cell death in chronic myelogenous leukemia cells via JNK-mediated p62/SQSTM1 expression and AMPK activation. Cancer Res 2010; 70: 1042-52.

23 Liu Y, Tseng M, Perdreau SA, Rossi F, Antonescu C, Besmer P, et al. Histone $\mathrm{H} 2 \mathrm{AX}$ is a mediator of gastrointestinal stromal tumor cell apoptosis following treatment with imatinib mesylate. Cancer Res 2007; 67: 2685-92.

24 Zhang YJ, Lu CR, Cao Y, Luo Y, Bao RF, Yan S, et al. Imatinib induces H2AX phosphorylation and apoptosis in chronic myelogenous leukemia cells in vitro via caspase-3/Mst1 pathway. Acta Pharmacol Sin 2012; 33: 551-7.

25 Dong Y, Xiong M, Duan L, Liu Z, Niu T, Luo Y, et al. H2AX phosphoryla- tion regulated by p38 is involved in Bim expression and apoptosis in chronic myelogenous leukemia cells induced by imatinib. Apoptosis 2014; 19: 1281-92.

26 Lu C, Zhu F, Cho YY, Tang F, Zykova T, Ma WY, et al. Cell apoptosis: requirement of $\mathrm{H} 2 \mathrm{AX}$ in DNA ladder formation, but not for the activation of caspase-3. Mol Cell 2006; 23: 121-32.

27 Lu C, Xiong M, Luo Y, Li J, Zhang Y, Dong Y, et al. Genome-wide transcriptional analysis of apoptosis-related genes and pathways regulated by H2AX in lung cancer A549 cells. Apoptosis 2013; 18: 1039-47.

28 Milella M, Kornblau SM, Estrov Z, Carter BZ, Lapillonne H, Harris D, et al. Therapeutic targeting of the MEK/MAPK signal transduction module in acute myeloid leukemia. J Clin Invest 2001; 108: 851-9.

29 Banerjee Mustafi S, Chakraborty PK, Raha S. Modulation of Akt and ERK1/2 pathways by resveratrol in chronic myelogenous leukemia $(\mathrm{CML})$ cells results in the downregulation of Hsp70. PLoS One 2010; 5: e8719. 